\title{
Hepatic Arterial Configuration in Relation to the Segmental Anatomy of the Liver; Observations on MDCT and DSA Relevant to Radioembolization Treatment
}

\author{
Andor F. van den Hoven - Maarten S. van Leeuwen • \\ Marnix G. E. H. Lam • Maurice A. A. J. van den Bosch
}

Received: 30 October 2013/Accepted: 27 January 2014/Published online: 7 March 2014

(C) Springer Science+Business Media New York and the Cardiovascular and Interventional Radiological Society of Europe (CIRSE) 2014

\begin{abstract}
Purpose Current anatomical classifications do not include all variants relevant for radioembolization (RE). The purpose of this study was to assess the individual hepatic arterial configuration and segmental vascularization pattern and to develop an individualized RE treatment strategy based on an extended classification.

Methods The hepatic vascular anatomy was assessed on MDCT and DSA in patients who received a workup for RE between February 2009 and November 2012. Reconstructed MDCT studies were assessed to determine the hepatic arterial configuration (origin of every hepatic arterial branch, branching pattern and anatomical course) and the hepatic segmental vascularization territory of all branches. Aberrant hepatic arteries were defined as hepatic arterial branches that did not originate from the celiac axis/CHA/PHA. Early branching patterns were defined as hepatic arterial branches originating from the celiac axis/CHA.

Results The hepatic arterial configuration and segmental vascularization pattern could be assessed in 110 of 133 patients. In 59 patients (54\%), no aberrant hepatic arteries or early branching was observed. Fourteen patients without
\end{abstract}

\footnotetext{
A. F. van den Hoven $(\bowtie) \cdot$ M. S. van Leeuwen

M. G. E. H. Lam - M. A. A. J. van den Bosch

Department of Radiology and Nuclear Medicine, University

Medical Center Utrecht, Room E.01.132, Heidelberglaan 100,

3584 CX Utrecht, The Netherlands

e-mail: a.f.vandenhoven@umcutrecht.nl

M. S. van Leeuwen

e-mail: m.s.vanleeuwen@umcutrecht.nl

M. G. E. H. Lam

e-mail: m.lam@umcutrecht.nl

M. A. A. J. van den Bosch

e-mail: mbosch@umcutrecht.nl
}

aberrant hepatic arteries (13\%) had an early branching pattern. In the 37 patients (34\%) with aberrant hepatic arteries, five also had an early branching pattern. Sixteen different hepatic arterial segmental vascularization patterns were identified and described, differing by the presence of aberrant hepatic arteries, their respective vascular territory, and origin of the artery vascularizing segment four.

Conclusions The hepatic arterial configuration and segmental vascularization pattern show marked individual variability beyond well-known classifications of anatomical variants. We developed an individualized RE treatment strategy based on an extended anatomical classification.

Keywords Interventional oncology $\cdot$ Liver/Hepatic . Radioembolization

\section{Introduction}

The extensive research of the past has provided insight in the large individual variability in the origin of the hepatic arteries [1-6]. However, current classifications of variant hepatic arterial anatomy do not include all variants relevant for hepatic radioembolization (RE), such as early branching patterns, a variable origin of the artery vascularizing segment 4 (S4), and the vascular territory of aberrant hepatic arteries. Correct identification of these variants may have a significant impact on treatment strategy, including coil embolization of aberrant arteries, planning the number of injection positions, and pretreatment activity calculation.

Both the origin and the vascular territory of the hepatic arteries differ notably in the embryological from the adult liver. In the early stages of hepatic formation, an embryological left hepatic artery (eLHA) originates from the left gastric artery (LGA) and supplies S2, whilst an eRHA 
originates from the superior mesenteric artery (SMA) and vascularizes segments 6-7. The eMHA originates from the celiac axis and vascularizes the remaining $\mathrm{S} 3,4,5,8$ of the liver [7, 8]. Eventually, the eLHA and eRHA regress and the eMHA persists, resulting in a normal hepatic arterial configuration of the adult liver, in which the celiac axis gives rise to a left (LHA) and right hepatic artery (RHA). When the eLHA or eRHA fails to regress, one or more aberrant hepatic arteries persists.

Aberrant hepatic arteries, defined as hepatic arterial branches that do not originate from the celiac axis, common (CHA) or proper hepatic artery (PHA), are common (reported prevalence 21-45\%) [1-5]. Frequently encountered variants are aberrant LHAs originating from the LGA and aberrant RHA's originating from the SMA. According to the early work of Michels, aberrant hepatic arteries can be further divided into accessory and replaced hepatic arteries, both with a unique territory of blood-supply within the liver [1]. A replaced hepatic artery is a full substitute for the LHA or RHA originating from the celiac axis. An accessory hepatic artery, on the other hand, complements its normally derived counterpart, and only supplies a part of the right or left hemi-liver $[1,9]$.

To the best of our knowledge, the relationship between the individual arterial branches and their corresponding arterial segmental territories has not been described for patients with aberrant hepatic arteries. Therefore, the vascular territory of accessory hepatic arteries remains unknown. The combination of aberrant hepatic arteries and a variable origin of the artery vascularizing S4 can result in highly variable individual patterns of segmental hepatic arterial vascularization that may require different treatment strategies [10]. Technological advances enable an accurate pretreatment assessment of the hepatic arterial anatomy on multidetector computed tomography (MDCT). Consequently, a treatment strategy, tailored to the patient's individual anatomy, can be determined before the pretreatment angiography.

The purpose of this study was to assess the individual hepatic arterial configuration (presence of aberrant hepatic arteries and order of arterial branching) and the arterial segmental vascularization pattern on triphasic MDCT and digital subtraction angiography (DSA) in patients receiving a workup for RE. Furthermore, we developed an individualized RE treatment strategy based on an extended classification of variant hepatic arterial anatomy.

\section{Patients and Methods}

Patients

All patients who were evaluated for RE between February 2009 and November 2012 in our institute were included in this study. These patients were all diagnosed with primary or metastatic liver cancer and received a triphasic liver MDCT and standard pretreatment angiography specifically to assess the eligibility for RE. During the angiography, DSA of the hepatic arteries was obtained (Allura Xper FD 20, Philips Medical Systems, Best, The Netherlands) and technetium99m-labelled macro-albumin aggregates $\left({ }^{99 \mathrm{~m}} \mathrm{Tc}-\mathrm{MAA}\right.$, TechneScan LyoMaa, Mallinckrodt Medical, Petten, The Netherlands) were administered to assess the eligibility for RE. Exclusion criteria for this study were: prior extensive liver surgery, nonassessable anatomy of the hepatic vasculature due to severe cirrhotic liver disease or massive tumor involvement. These data also were used in another study [11]. Our institutional ethics committee waived the need for informed consent for this retrospective study.

Imaging Protocol

A 16-, 64-, or 256-detector row CT scanner (MX 8000; Brilliance 64; Brilliance iCT; Philips Medical Systems, Eindhoven, The Netherlands) was used to obtain triphasic MDCT images. Alternatively, in some patients the triphasic liver CT scan was acquired on an integrated positron emission tomography-computed tomography system (Biograph $^{\mathrm{TM}} \mathrm{mCT}$, Siemens Healthcare, Erlangen, Germany). After injection of intravenous contrast, hepatic arterial, venous and equilibrium phase images were obtained with a post-threshold (aorta, $>100 \mathrm{HU}$ ) delay of 20, 55, and $300 \mathrm{~s}$ respectively. All patients received 11 of oral diluted water-soluble contrast agent (Telebrix Gastro, $300 \mathrm{mgl} / \mathrm{ml}$ ). Depending on body weight, 150 or $200 \mathrm{ml}$ of contrast (Iopromide $300 \mathrm{mgl} / \mathrm{ml}$; Ultravist, Bayern Schering Pharma AG, Berlin, Germany) was injected in an anticubital vein, at a rate of $5 \mathrm{ml} / \mathrm{s}$.

Image Interpretation

CT images were analyzed on a workstation (Intellispace Portal; Philips Medical Systems). First, the portal phase was reviewed to determine the schematic liver segmentation, based on Couinaud's classification of segmental liver anatomy. To this purpose, the left, middle, and right hepatic veins were used to indicate the vertical scissurae, and a horizontal plane through the right portal trunk was assumed to indicate the transverse scissura.

Subsequently, axial and coronal maximum intensity projections of the arterial phase were reviewed to determine the origin of all hepatic arterial branches and follow their course up to their segmental territory of the liver. We named this the hepatic arterial segmental vascularization pattern (Table 1).

The artery vascularizing $\mathrm{S} 4$ was named a middle hepatic artery (MHA) when originating from the CHA/PHA as a 
Table 1 Terminology

\begin{tabular}{|c|c|}
\hline Term & Definition \\
\hline $\begin{array}{l}\text { Standard hepatic arterial } \\
\text { configuration }\end{array}$ & $\begin{array}{l}\text { No early branching pattern and no } \\
\text { aberrant hepatic arteries }\end{array}$ \\
\hline $\begin{array}{l}\text { Variant hepatic arterial } \\
\text { configuration }\end{array}$ & $\begin{array}{l}\text { Early branching pattern of the hepatic } \\
\text { arteries and/or aberrant hepatic arteries }\end{array}$ \\
\hline Early branching pattern & $\begin{array}{l}\text { Trifurcation of the CHA (i.e., absence of } \\
\text { the PHA), early branching LHA and/or } \\
\text { RHA from the CHA (proximal to the } \\
\text { origin of the GDA) or LHA and/or RHA } \\
\text { originating directly from the celiac axis }\end{array}$ \\
\hline Aberrant hepatic artery & $\begin{array}{l}\text { Aberrantly derived hepatic artery, not } \\
\text { originating from the celiac axis, CHA or } \\
\text { PHA. Aberrant hepatic arteries can be } \\
\text { divided into accessory and replaced } \\
\text { hepatic arteries }\end{array}$ \\
\hline Accessory hepatic artery & $\begin{array}{l}\text { Aberrant hepatic artery that vascularizes } \\
\text { the left (S2) or right hemi-liver partially } \\
\text { (any segments), existing in addition to a } \\
\text { normally derived LHA and RHA }\end{array}$ \\
\hline Replaced hepatic artery & $\begin{array}{l}\text { Aberrant hepatic artery that vascularizes } \\
\text { the left (S2-3 or S2-4) or right hemi- } \\
\text { liver (S5-8). An rCHA exists when the } \\
\text { entire hepatic trunk is replaced to } \\
\text { another source than the celiac axis }\end{array}$ \\
\hline $\begin{array}{l}\text { Arterial segmental } \\
\text { vascularization pattern }\end{array}$ & $\begin{array}{l}\text { Hepatic segmental estuaries of the } \\
\text { individual hepatic arteries }\end{array}$ \\
\hline Segment 4 artery (A4) & $\begin{array}{l}\text { Hepatic arterial branch vascularizing S4, } \\
\text { and originating from the LHA and/or } \\
\text { RHA }\end{array}$ \\
\hline $\begin{array}{l}\text { Middle hepatic artery } \\
\text { (MHA) }\end{array}$ & $\begin{array}{l}\text { Hepatic arterial branch vascularizing S4, } \\
\text { and originating directly from the CHA } \\
\text { or PHA }\end{array}$ \\
\hline
\end{tabular}

The definitions for the anatomical terminology used in this manuscript are summarized in this table. The definitions for aberrant hepatic arteries, replaced, and accessory hepatic arteries are in accordance with the work by Michels [1]. The distinction between A4 and MHA is based on the definition used by Wang et al. [12]. The definitions for hepatic arterial configuration, arterial segmental vascularization pattern, early branching pattern, and specification of segments that can be vascularized by replaced and accessory left hepatic arteries were created as part of the extended anatomical classification of variant hepatic arterial anatomy described in this manuscript

hilar artery, and a S4 artery (A4) when originating as an intrahepatic branch from the LHA and/or RHA [12]. Aberrant hepatic arteries were defined as hepatic arterial branches that did not originate from the celiac axis directly, or indirectly through the CHA or PHA [1]. Aberrant hepatic arteries were further classified into accessory and replaced hepatic arteries. We also assessed the branching pattern of the CHA. The CHA was defined as the hepatic arterial branch between the celiac axis and the gastroduodenal artery (GDA). Early branching patterns were defined as a trifurcation of the CHA (i.e., absence of PHA), early branching of the LHA and/or RHA from the CHA (proximal to the origin of the GDA), or LHA and/or RHA originating directly from the celiac axis. The combination of the origin of the hepatic arteries and the branching pattern of the CHA was termed the hepatic arterial configuration. In the absence of aberrant hepatic arteries and early branching patterns, patients were classified as having a standard hepatic arterial configuration.

After review of the MDCT images, DSA-images of the hepatic arterial vasculature in the frontal plane were reviewed to confirm the order of arterial branching. All imaging was assessed by two readers (AvdH and MSvL) in consensus.

\section{Statistics}

Descriptive statistics were performed with the use of SPSS software version 20 for windows (IBM SPSS Statistics, Chicago, IL). Percentages were rounded to the nearest whole number.

\section{Results}

\section{Patients}

Of 133 patients who received a workup for RE with MDCT and DSA, 19 (14\%) were excluded for the following reasons: previous extensive liver surgery $(n=12)$, severe liver cirrhosis distorting the liver anatomy $(n=4)$, or large tumor mass distorting the liver anatomy $(n=3)$. The hepatic arterial configuration and arterial segmental vascularization of the remaining patients were assessed (Fig. 1). However, in four patients (4\%), the arterial segmental vascularization could not be assessed reliably on MDCT, due to the very small caliber of the arterial branches. Consequently, we present the results of the hepatic arterial configuration and arterial segmental vascularization of the remaining 110 patients.

\section{Hepatic Arterial Configuration and Segmental Vascularization Pattern}

Fifty-nine (54\%) patients had a standard hepatic arterial configuration, without aberrant arteries and/or early branching, i.e., standard hepatic arterial anatomy. Fourteen patients $(13 \%)$ had normally derived hepatic arteries, but one or more arteries with an early branching pattern (Table 2).

In 37 patients (34\%), a variant origin of at least one hepatic artery was present (Table 3). All aberrant LHAs originating from the LGA coursed upward in the cranial part of the lesser omentum and entered the hilar plate through the fissure for the ligamentum venosum, before 
Fig. 1 Patient inclusion

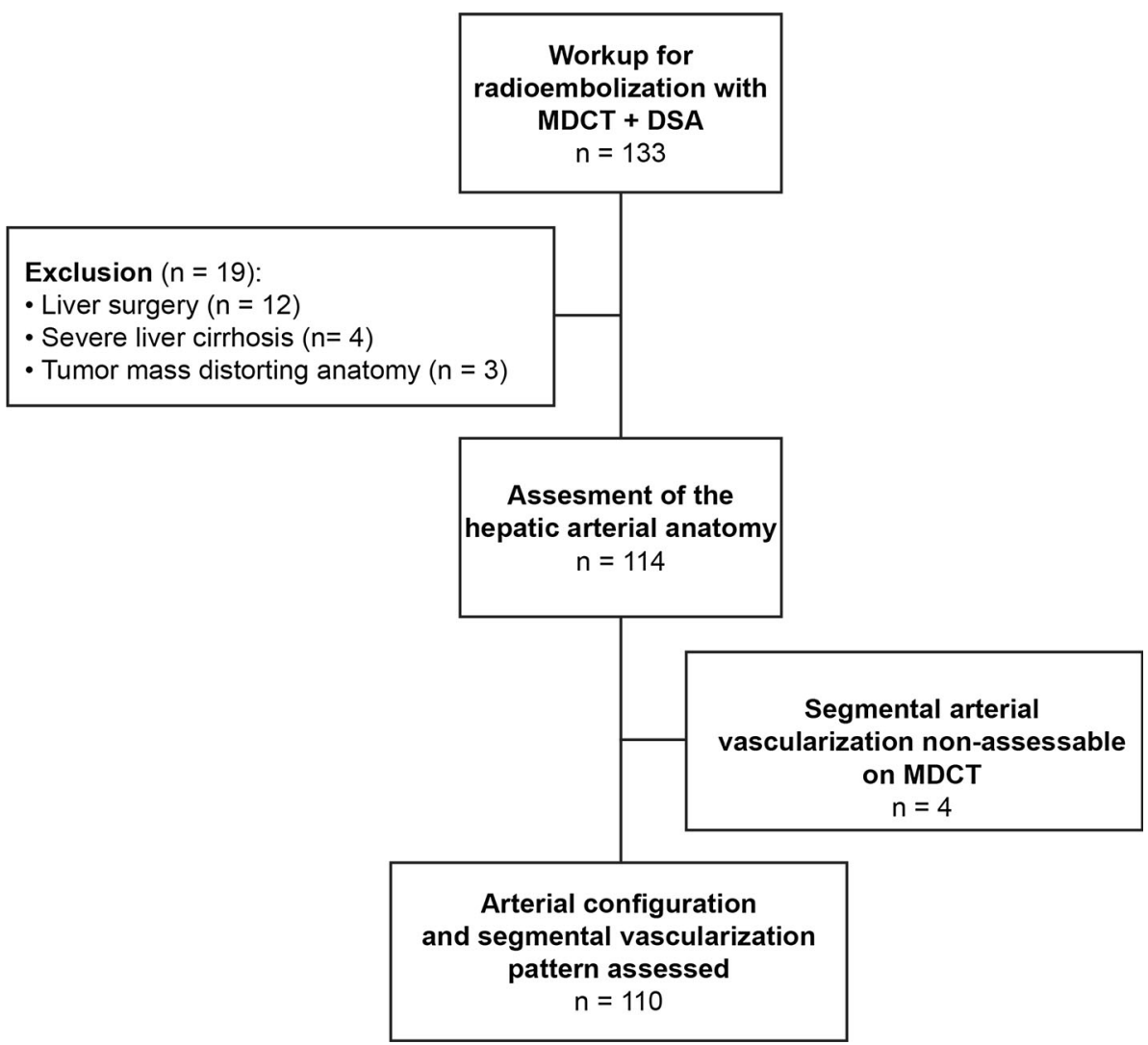

Table 2 Observed early branching patterns

\begin{tabular}{ll}
\hline Early branching pattern & $\begin{array}{l}\text { Prevalence } \\
(\%)\end{array}$ \\
\hline $\begin{array}{l}\text { Early branching patterns in patients without aberrant } \\
\text { hepatic arteries }\end{array}$ & $14(13 \%)$ \\
Trifurcation CHA in GDA, LHA and RHA & $9(8 \%)$ \\
Early branching LHA from CHA & $2(2 \%)$ \\
Early branching RHA from CHA & $1(1 \%)$ \\
Early branching LHA and RHA from CHA & $1(1 \%)$ \\
RHA originating directly from CA & $1(1 \%)$ \\
Early branching patterns in patients with aberrant & $5(5 \%)$ \\
hepatic arteries & $2(2 \%)$ \\
rRHA (SMA) and trifurcation CHA in GDA, LHA & \\
and MHA & $1(1 \%)$ \\
rRHA (SMA) and early branching LHA from CHA & $1(1 \%)$ \\
rLHA (LGA) and RHA originating directly from CA & $1(1 \%)$ \\
rCHA (SMA) and early branching RHA from CHA & $1 \%$
\end{tabular}

Early branching patterns (in patients without and with aberrant hepatic arteries) are displayed

CHA common hepatic artery, GDA gastroduodenal artery, LHA left hepatic artery, $R H A$ right hepatic artery, $M H A$ middle hepatic artery, $r R H A$ replaced right hepatic artery, $r L H A$ replaced left hepatic artery, rCHA replaced common hepatic artery, SMA superior mesenteric artery, $L G A$ left gastric artery, $C A$ celiac axis
Table 3 Observed aberrant hepatic arteries

\begin{tabular}{llrl}
\hline $\begin{array}{l}\text { Variant } \\
\text { configuration }\end{array}$ & Origin & Prevalence $(\%)$ & $\begin{array}{l}\text { Michels/Hiatt } \\
\text { classification }\end{array}$ \\
\hline $\begin{array}{l}\text { No aberrant hepatic } \\
\text { arteries }\end{array}$ & - & $73(66 \%)$ & Type I/I \\
rLHA & LGA & $9(8 \%)$ & Type II/II \\
rRHA & SMA/Aorta & $16(15 \%) / 1(1 \%)$ & Type III/III \\
rLHA + rRHA & LGA/SMA & $3(3 \%)$ & Type IV/IV \\
aLHA & LGA & $1(1 \%)$ & Type V/II \\
aRHA & SMA/GDA & $0(0 \%) / 1(1 \%)$ & Type VI/III \\
aLHA + aRHA & LGA/SMA & $1(1 \%)$ & Type VII/IV \\
aLHA + rRHA & LGA/SMA & $1(1 \%)$ & Type VIII/IV \\
rLHA + aRHA & LGA/SMA & $1(1 \%)$ & Type VIII/IV \\
rCHA & SMA & $3(1 \%)$ & Type IX/V \\
\hline
\end{tabular}

Observed configurations of aberrantly derived hepatic arteries are displayed with the origin of the accessory or replaced hepatic artery, and observed prevalence denoted in number of patients (percentage of total). The Michels and Hiatt classification types are displayed to allow for comparison

$r L H A$ replaced left hepatic artery, aLHA accessory left hepatic artery, $r R H A$ replaced right hepatic artery, aRHA accessory right hepatic artery, $r C H A$ replaced common hepatic artery, $L G A$ left gastric artery, SMA superior mesenteric artery, GDA gastroduodenal artery 
continuing their course alongside the left portal trunk within the umbilical fissure, to reach their respective segmental territory of liver parenchyma. All aberrant RHAs originating from the SMA displayed a retroportal course within the hepatoduodenal ligament, traversing between the main portal vein and inferior vena cava, before entering the hilar plate. Four out of five $(80 \%)$ early branching RHA's (including one RHA originating directly from the celiac axis) displayed a similar retroportal course.

The hepatic arterial segmental vascularization patterns are summarized in Table 4 and schematically illustrated in Figs. 2, 3, 4, 5 and 6. An example of DSA and multiplanar MDCT images of the hepatic arterial segmental vascularization pattern is shown in Fig. 7 for a patient with an rRHA originating from the SMA.

\section{Discussion}

In this study, we assessed the origin, branching pattern, course, and segmental vascularization pattern of the hepatic arteries on triphasic liver MDCT and DSA in 110 patients receiving a workup for RE. The prevalence and configurations of aberrant hepatic arteries (Table 3) and early branching patterns (Table 2) were comparable to those previously published [1-6]. Sixteen different hepatic arterial segmental vascularization patterns were identified, differing by the presence of accessory or replaced hepatic arteries, their respective vascular territory, and the origin of artery vascularizing $\mathrm{S} 4$. This marked variability reflects the complex nature of the arterial liver vascularization, justifying a detailed pre-procedural assessment of the individual hepatic arterial configuration and segmental vascularization pattern prior to RE.

The extensive research conducted in the past five decades has greatly enhanced our knowledge of variant hepatic arterial anatomy [1-6, 13]. In 1966, Michels introduced an internationally recognized classification of hepatic arterial variations, in which he described ten types of arterial configuration, based on his observations in 200 autopsy dissections [1]. Later, Hiatt et al. introduced a reduced classification with six types, based on a review of operative

Table 4 Segmental hepatic arterial vascularization patterns

\begin{tabular}{|c|c|c|c|c|}
\hline Originating from the celiac axis & Originating from the SMA & Originating from the LGA & Prevalence & Figure \\
\hline No aberrant hepatic arteries & & & $73(66 \%)$ & Fig. 2 \\
\hline LHA [2-4] \& RHA [5-8] & - & - & $51(46 \%)$ & Fig. $2 \mathrm{a}$ \\
\hline LHA [2-3] \& RHA [4-8] & - & - & $21(19 \%)$ & Fig. $2 b$ \\
\hline LHA [2-3], MHA [4] \& RHA [5-8] & - & - & $1(1 \%)$ & Fig. 2c \\
\hline Aberrant left hepatic arteries & & & $10(9 \%)$ & Fig. 3 \\
\hline RHA [4-8] & - & rLHA [2-3] & $7(6 \%)$ & Fig. 3a \\
\hline RHA [5-8] & - & rLHA [2-4] & $2(2 \%)$ & Fig. $3 b$ \\
\hline LHA [3-4] \& RHA [5-8] & - & aLHA [2] & $1(1 \%)$ & Fig. $3 \mathrm{c}$ \\
\hline Aberrant right hepatic arteries & & & $18(17 \%)$ & Fig. 4 \\
\hline LHA [2-4] & rRHA $[5-8]^{\mathrm{a}}$ & - & $15(14 \%)$ & Fig. $4 \mathrm{a}$ \\
\hline LHA [2-3] & rRHA [4-8] & - & $1(1 \%)$ & Fig. $4 \mathrm{~b}$ \\
\hline LHA [2-4] & rRHA [4-8] & - & $1(1 \%)$ & Fig. $4 \mathrm{c}$ \\
\hline LHA [2-3], RHA $[4,6-8] \&$ aRHA $[5]^{\mathrm{b}}$ & & - & $1(1 \%)$ & Fig. $4 d$ \\
\hline Aberrant right and left hepatic arteries & & & $6(6 \%)$ & Fig. 5 \\
\hline MHA [4] & rRHA [5-8] & rLHA [2-3] & $2(2 \%)$ & Fig. 5a \\
\hline- & rRHA [5-8] & rLHA [2-4] & $1(1 \%)$ & Fig. $5 b$ \\
\hline LHA [3-4] \& RHA [7-8] & aRHA [5-6] & aLHA [2] & $1(1 \%)$ & Fig. $5 \mathrm{c}$ \\
\hline LHA [3-4] & rRHA [5-8] & aLHA [2] & $1(1 \%)$ & Fig. $5 d$ \\
\hline RHA [7] & aRHA $[5,6,8]$ & rLHA [2-4] & $1(1 \%)$ & Fig. $5 \mathrm{e}$ \\
\hline Replaced common hepatic artery & & & $3(3 \%)$ & Fig. 6 \\
\hline- & LHA [2-4] \& RHA [5-8] & - & $3(3 \%)$ & Fig. 6 \\
\hline
\end{tabular}

The segmental arterial vascularization patterns are summarized in this table. The main arterial branches are followed by their segmental territory of vascularization between brackets

LHA left hepatic artery, RHA right hepatic artery, MHA middle hepatic artery, aRHA/aLHA accessory RHA/LHA, $r R H A / L H A$ replaced RHA/ LHA

${ }^{\text {a }}$ In one patient the rRHA originated from the aorta instead of the SMA

b aRHA originating from the GDA 
Fig. 2 A-C Schematic representation of the hepatic arterial vascularization pattern in patients without aberrant hepatic arteries. (The illustrative concept of Figs. 2, 3, 4, 5 and 6 is reprinted from Jin et al., Anatomical variations of the origin of the segment 4 hepatic artery and their clinical implications. Liver

Transplantation 2008;14:1180-1184.)

\section{No aberrant hepatic arteries}
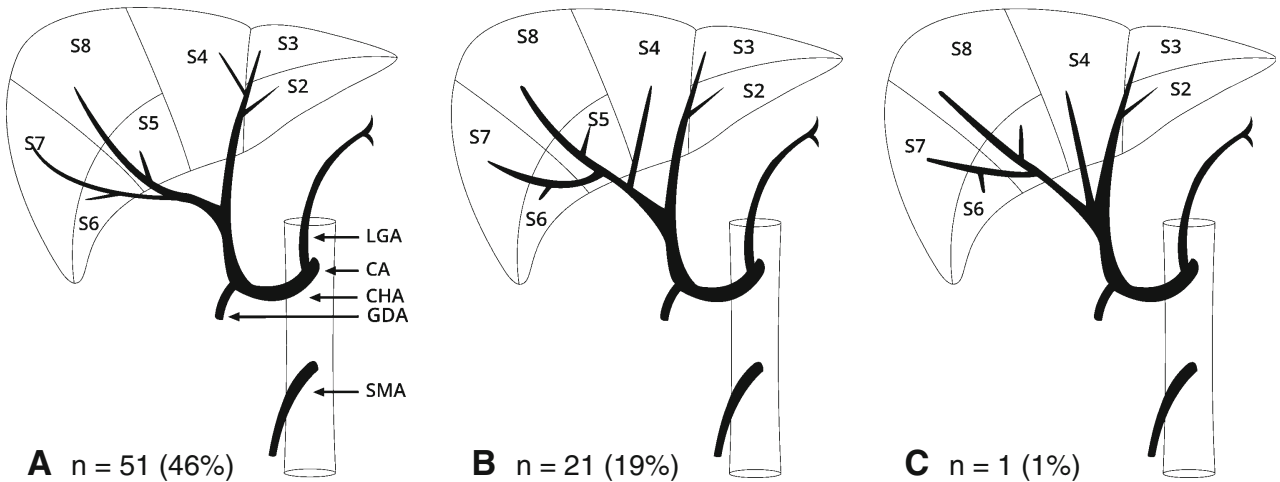

Fig. 3 A-C Schematic representation of the hepatic arterial vascularization pattern in patients with aberrant left hepatic arteries

\section{Aberrant left hepatic arteries}

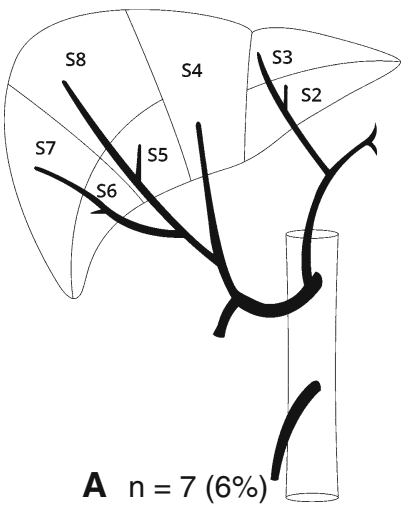

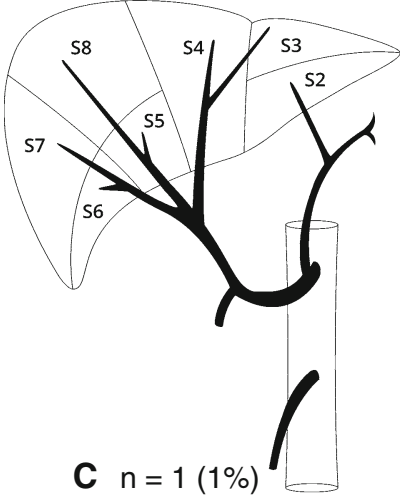

C $\mathrm{n}=1(1 \%)$ reports of 1,000 patients who underwent liver harvesting for orthotopic transplantation. In this adaptation, the distinction between replaced and accessory hepatic arteries was abandoned [2]. Subsequent studies on this subject described the prevalence of aberrant hepatic arteries in a large number of patients, based on surgical dissection [5], angiography $[3,4,6]$ and later also CT angiography [1417], and extended Michels' and Hiatt's classifications with early branching patterns and rare variants. However, none of these studies included the origin of the artery vascularizing S4 and the vascular territory of aberrant hepatic arteries in their overview of variant hepatic arterial anatomy. This may be attributable to the fact that only CT imaging can provide a complete overview on the origin, branching pattern, and segmental vascularization of the hepatic arterial branches. Our study, therefore, is the first to provide a comprehensive overview of the hepatic arterial segmental vascularization patterns in patients with and without aberrant hepatic arteries.

In the 73 patients without aberrant hepatic arteries, we identified three vascularization patterns, differing by the origin of the artery vascularizing S4 (Fig. 2). Studies that previously investigated the origin of this arterial branch,

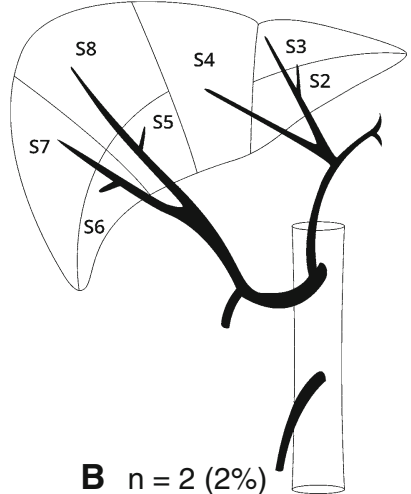

often used different definitions, and terms, such as A4 and MHA were interchangeably used [8, 12, 18]. From an anatomical point of view, the term MHA refers to a hilar artery that originates from the CHA or PHA, between the origins of the LHA and RHA, whereas A4 also can refer to a small intrahepatic branch that originates from the distal LHA or RHA [12]. Previous studies showed contradictory results, which may be attributable to these differences in definition. Two recent studies indicated that the artery vascularizing S4 predominantly originated from the RHA and less frequently from the LHA, from both hepatic arteries or directly from the PHA in a trifurcation $[8,12]$. Our results are more in agreement with earlier studies [1, 9, 18], suggesting that the artery vascularizing S4 predominantly originates from the LHA and less frequently from the RHA, both hepatic arteries or the PHA.

We identified various arterial segmental vascularization patterns in patients with aberrant hepatic arteries. Major differences were observed in the origin of the artery vascularizing S4 and the liver territory vascularized by the aberrant hepatic arteries. Wang et al. [12] suggested that in patients with a rLHA or rRHA, a MHA consistently arises from the CHA. Although this was true for the majority of 
Fig. 4 A-D Schematic representation of the hepatic arterial vascularization pattern in patients with aberrant right hepatic arteries
Aberrant right hepatic arteries
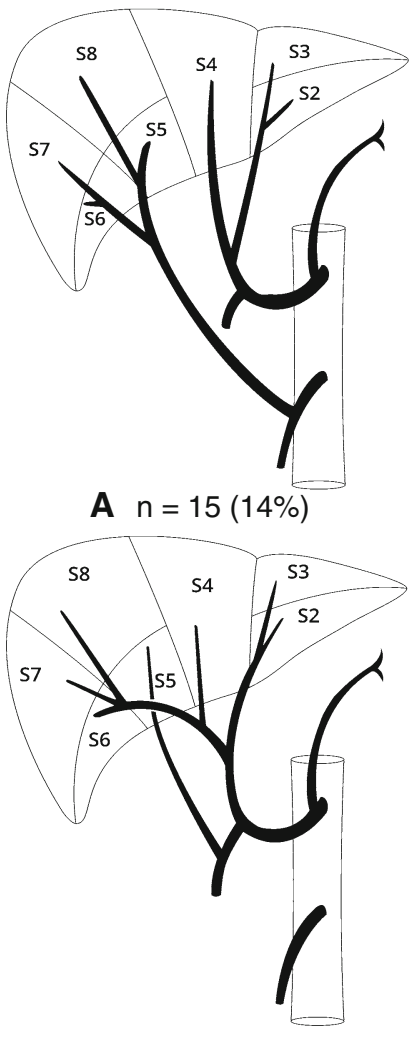

D $\mathrm{n}=1(1 \%)$

patients with a rLHA or rRHA in our study, we also found $\mathrm{A} 4 \mathrm{~s}$ originating from replaced hepatic arteries. One patient even had a double vascularization of $\mathrm{S} 4$, with one A4 originating from the rRHA and the other A4 originating from the LHA (Fig. 4C). Moreover, in patients with a combination of a rLHA and rRHA, S4 was vascularized by a MHA originating from the CHA in two patients (Fig. 5A) and by an A4 originating from the rLHA in one patient (Fig. 5B). Three hitherto unreported arterial vascularization patterns were identified in patients with aRHAs (Figs. 4D, 5C, and 5E). This emphasizes the importance of characterizing an aberrant hepatic artery as being accessory or replaced, defined by the extent of its territory of blood supply and coexistence of a normally derived LHA and RHA [1]. Due to the variable vascularization of S4, we believe that the distinction between an accessory and replaced LHA should not depend on the origin of the artery vascularizing S4. Hence, an aLHA vascularizes S2 only, and an rLHA may vascularize S2-3 or S2-4.

Early anatomical studies that investigated the spatial relation between the portal venous and hepatic venous vasculature in corrosion casts of the liver have founded the concept of functional liver anatomy [9, 19-23]. These studies revealed that the liver consists of a functional

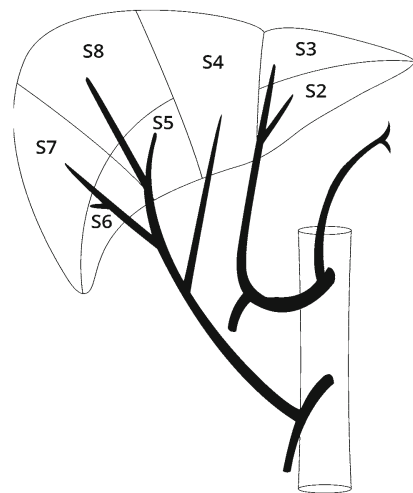

B $\mathrm{n}=1(1 \%)$

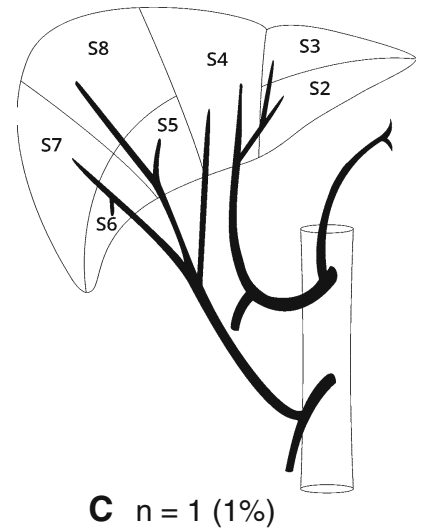

independent left and right hemiliver, divided by the main portal scissura. Later, Couinaud's concept of eight haemodynamically distinct liver segments, each with their own arterial and portal blood supply and biliary drainage, was adopted [7, 24]. Since the general acceptance of Couinaud's segmental anatomy model, the arterial segmental vascularization pattern has not received much scientific attention. This might be attributable to the recognition that the hepatic arterial branches are intimately related to the corresponding portal venous and biliary duct branches within the liver tissue and, therefore, display a similar course. However, this does not signify that the segmental vascularization pattern of the main hepatic arterial branches is equal to that of the major portal trunks that irrigate the, portal-venous defined, left and right hemiliver.

The treatment principle of intra-arterial RE is based on selective targeting of tumor invested liver tissue [25]. In current clinical practice, the calculated treatment activity of resin yttrium-90 microspheres (SIR-spheres ${ }^{\circledR}$, SIRTeX, Lane Cove, Australia) is based on the assumption that the LHA and RHA vascularize the entire left and right hemiliver respectively. Our findings suggest that this assumption is often not in agreement with anatomic reality, and that, in the presence of aberrant hepatic arteries, this method may lead to 
Fig. 5 A-E Schematic representation of the hepatic arterial vascularization pattern in patients with a combination of aberrant right and left hepatic arteries

\section{Aberrant left and right hepatic arteries}
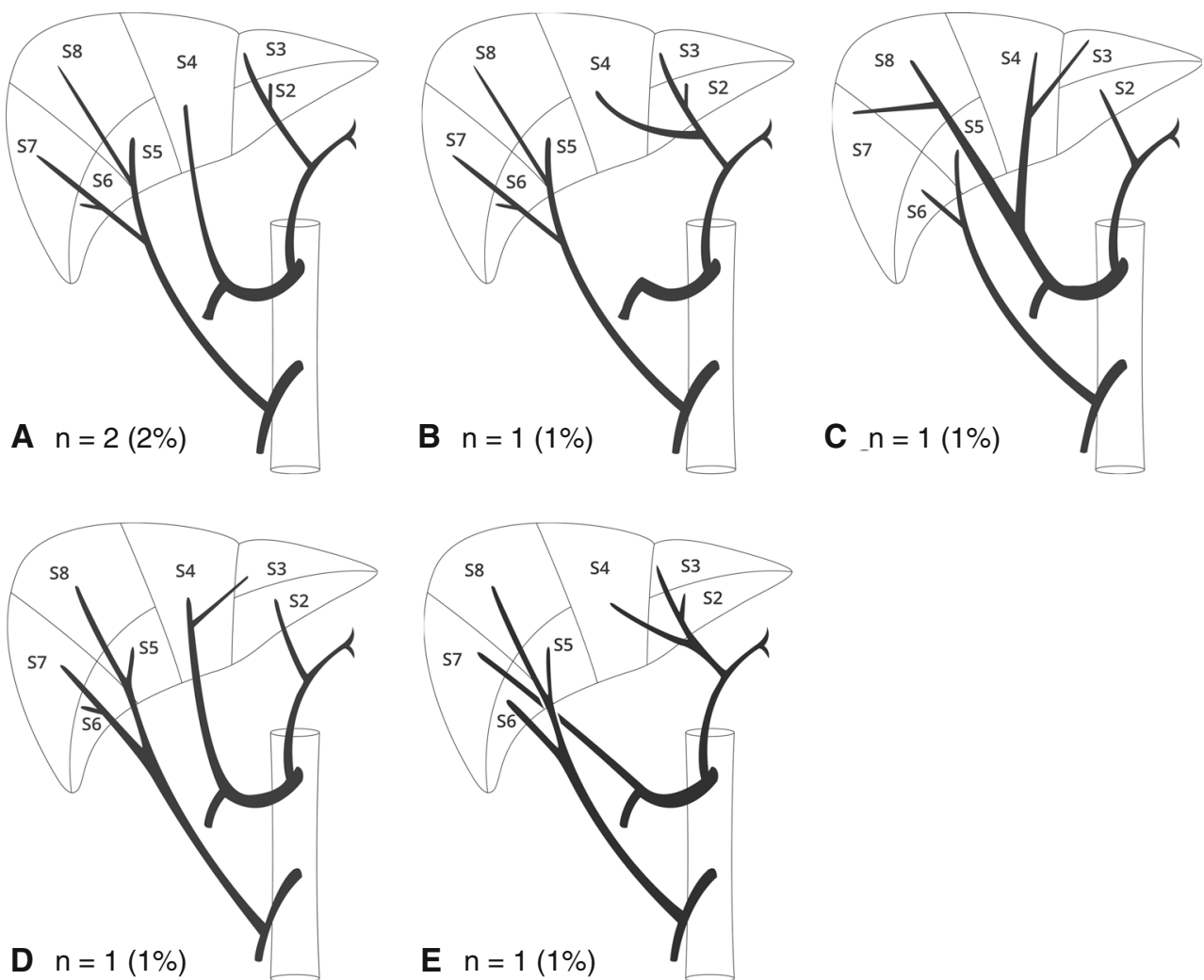

\section{Replaced CHA}

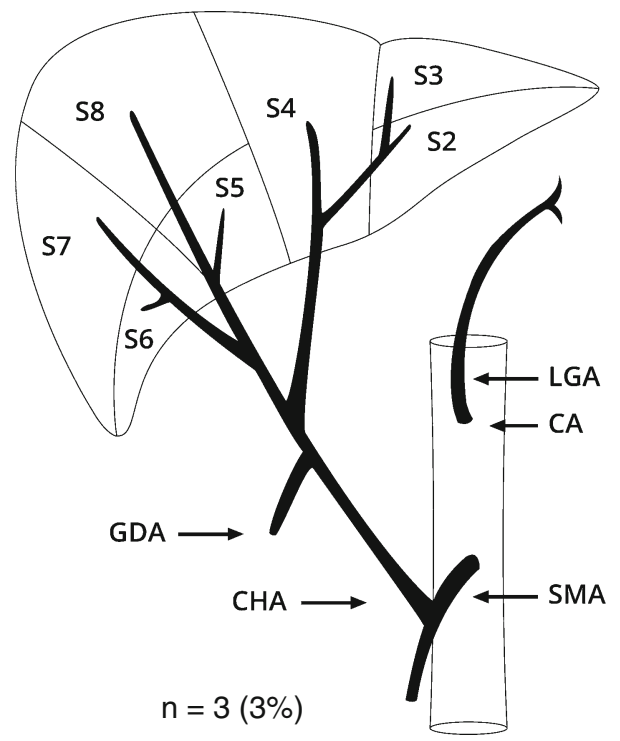

Fig. 6 Schematic representation of the hepatic arterial vascularization pattern in patients with a replaced common hepatic artery

erroneous results. Therefore, we advocate the utilization of personalized dosimetry for RE, based on the individual hepatic arterial vascularization pattern [26].

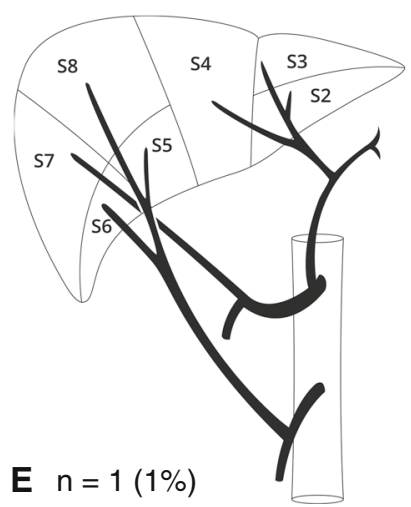

Following limitations of this study should be discussed. First, it was not our purpose to evaluate separately the diagnostic accuracy of MDCT and DSA in detecting aberrant hepatic arteries and early branching patterns. Instead, we used a combined approach of MDCT and DSA. DSA proved to be especially useful to determine the order of hepatic arterial branching in the area of the GDA, and to rule out the presence of a PHA when a trifurcation of the CHA was suspected on MDCT. Second, we could not assess the segmental arterial vascularization pattern in 4 of 114 patients $(4 \%)$, due to the small size of the hepatic arterial branches. Third, we deemed it unreliable to assess the origin of the arteries vascularizing S1. In the absence of tumor-induced hypertrophy, the small caliber of multiple feeders to $\mathrm{S} 1$ are beyond the resolution of current MDCT scanners. Fourth, we used Couinaud's model of segmental liver anatomy to describe the segmental arterial vascularization pattern. This model is a comprehensive topographical concept of functional liver anatomy that is easily interpretable and can be applied in most patients. However, an increasing body of evidence suggests that this concept may be an oversimplification [27-31]. Anatomical and radiological investigations have confirmed that the segmental boundaries, as indicated by the hepatic veins and the level of the right portal trunk, do 

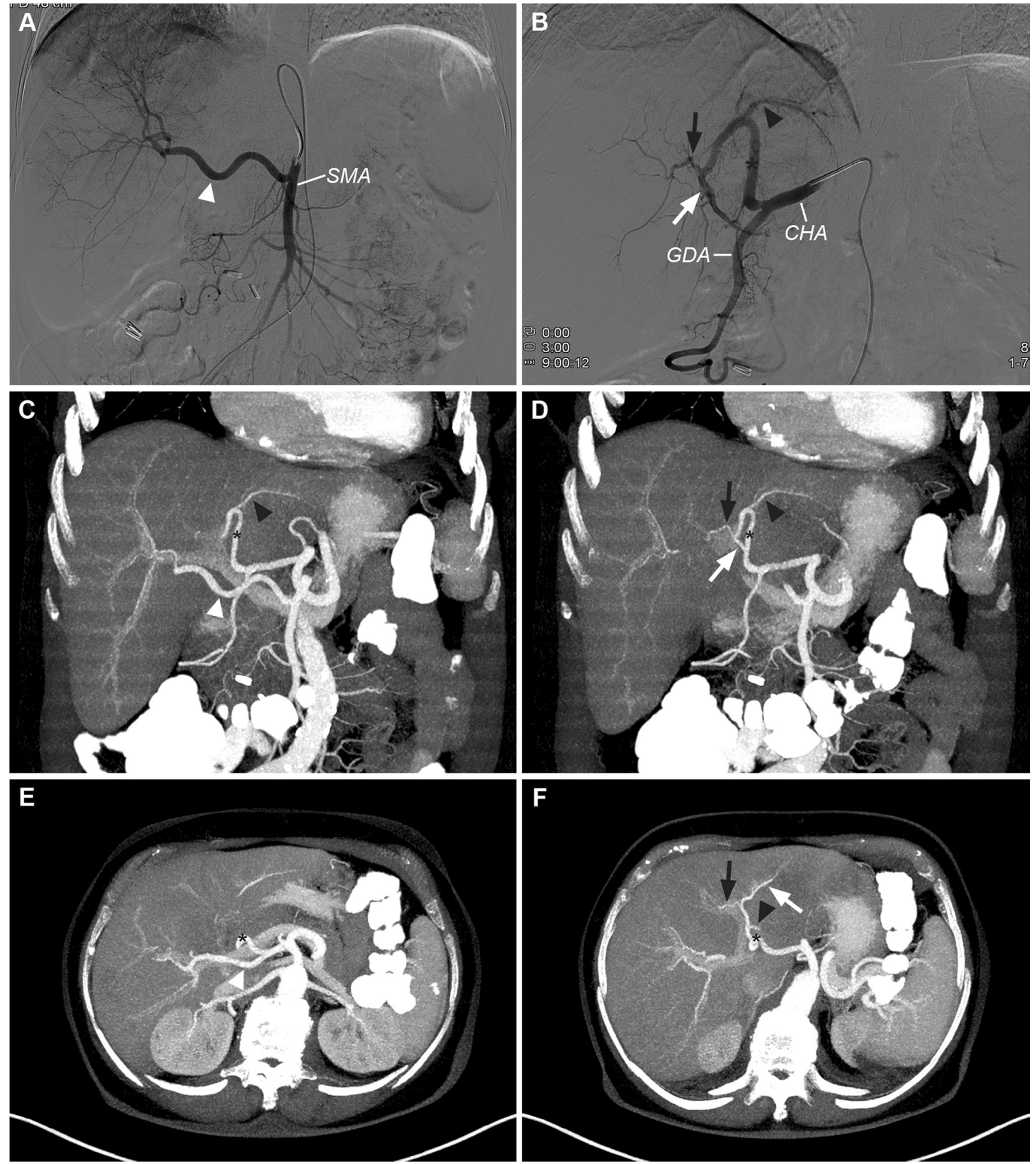

Fig. 7 DSA (A-B) and multiplanar MDCT (C-F) images of the hepatic arterial segmental vascularization pattern in a patient with an rRHA originating from the SMA. A Superior mesenteric arteriogram shows a replaced right hepatic artery (white arrowhead) originating from the SMA. B DSA obtained from the CHA. The CHA divides into GDA and LHA (black asterisk). The LHA first gives of a branch

to S2 (black arrowhead), and bifurcates more distally into a branch to S3 (white arrow) and S4 (black arrow). C-D Corresponding arterial phase coronal MIP images. E-F Corresponding arterial phase axial MIP images. Note the retroportal course of the rRHA (white arrowhead), and the perfect intrahepatic coordination between distal portal and arterial branches 


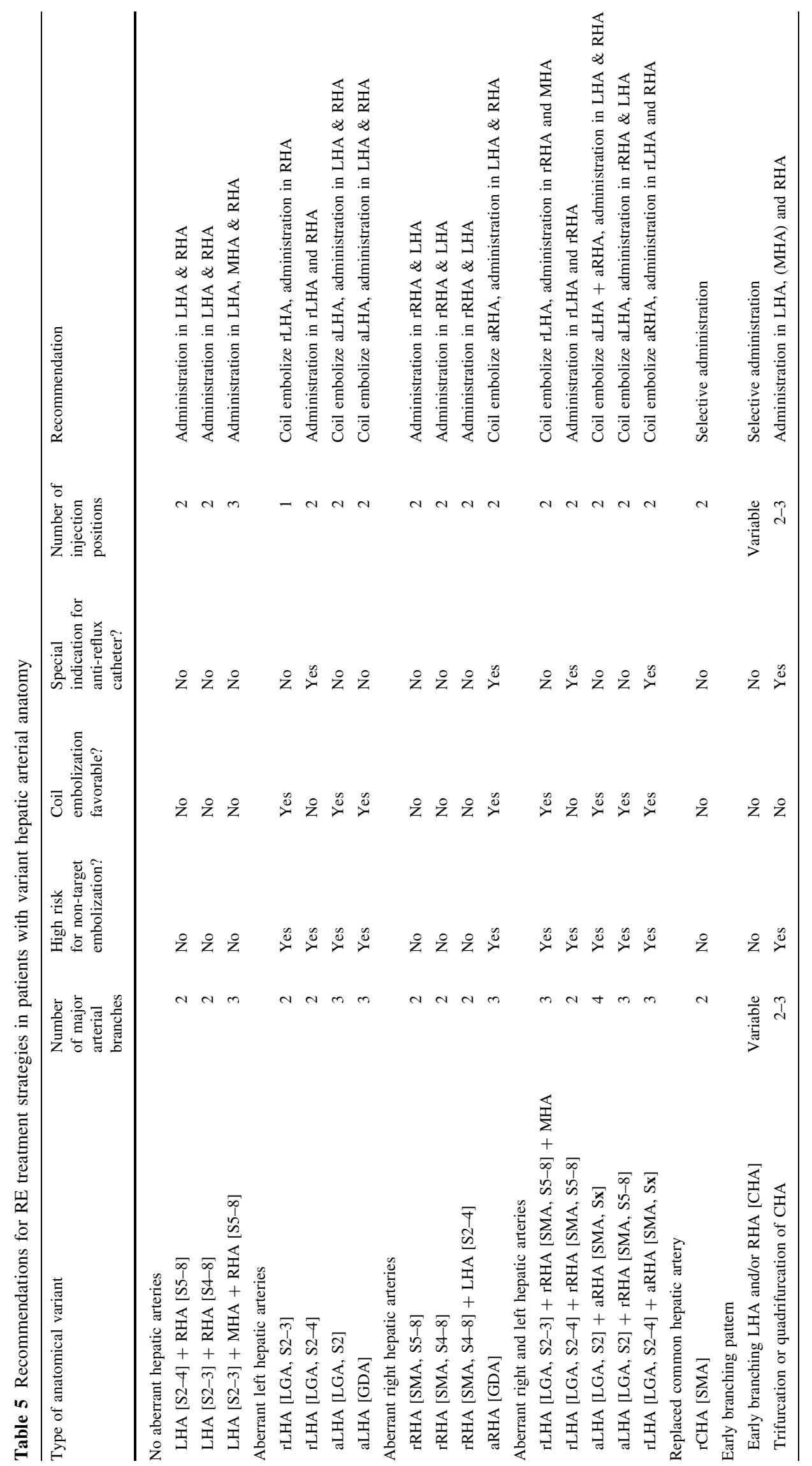


not consistently coincide with the exact location of the true portal scissurae in individual patients [28, 29, 31]. As a consequence, the true target volume of arterial branches can slightly differ from Couinaud's liver segments, which may have important implications for pretreatment dosimetry and the identification of tumor-feeding arteries. During the pretreatment angiography, C-arm cone beam computed tomography, a relatively new imaging modality integrated in modern angio-suites, may be used to delineate the exact territory of arterial perfusion through contrast-enhancement of the perfused liver parenchyma [32].

\section{Recommendations for an Individualized RE Treatment Strategy}

There is no consensus about how to deal with early branching patterns and aberrant hepatic arteries during RE. The ideal treatment strategy encompasses a safe, simple, and complete administration, with a low risk of nontarget embolization, few sites of selective administration, and adequate coverage of the target volume.

We developed an extended classification of variant hepatic arterial anatomy that can be used to characterize clinically relevant anatomical variants on MDCT before the pretreatment angiography and guide treatment planning. This MDCT-based assessment may reduce the procedural time, contrast, and radiation burden of the pretreatment angiography by superseding extensive mapping of the hepatic arterial anatomy with DSA. Furthermore, it may help to standardize treatment strategies in patients with complex anatomy and facilitate interdisciplinary communication with other physicians. We propose an individualized RE treatment strategy, based on the patients' type of variant anatomy (Table 5).

Various treatment strategies are feasible with regard to aberrant arteries. One strategy is to use aberrant hepatic arteries as a target vessel for selective administration with a standard endhole microcatheter. For the administration in branches at high risk for nontarget embolization, such as aberrant hepatic arteries originating from the LGA, an antireflux catheter, such as the Surefire Infusion System ${ }^{\circledR}$ (Surefire Medical Inc., Westminster, CO), may be used [33]. Another strategy is to coil embolize aberrant hepatic arteries to induce a redistribution of blood flow through small intrahepatic collateral pathways and administer microspheres in the hepatic artery that took over the coiled artery's vascular territory, resulting in a simplified and safe administration [34, 35].

In the absence of a direct comparison with regard to safety and treatment efficacy, neither of the strategies can be regarded as superior. After carefully weighing the risks and benefits for the different treatment strategies, we recommend coil embolization for aberrant hepatic arteries at increased risk for nontarget embolization that do not supply an entire hemiliver, such as an aLHA (vascularizing S2) or rLHA (vascularizing S2-3) originating from the LGA, and an accessory hepatic artery originating from the GDA (rare). Coil embolization also may be favorable for an aRHA originating from the SMA in patients who in addition have an aberrant left hepatic artery originating from the LGA. This can reduce significantly the complexity of the administration by limiting the number of selective administration sites. Other variants, such as rRHAs originating from the SMA, do not increase the risk for nontarget embolization and supply a large territory of the liver that may not be sufficiently vascularized by small intrahepatic collaterals to allow for complete passage of all infused resin microspheres [34]. They therefore are recommended to be used as target vessel. Replaced left hepatic arteries vascularizing S2-4 increase the risk for nontarget embolization but do supply a rather large part of the liver. An antireflux catheter may be used for selective administration in these variants. The latter strategy also can be applied in patients with a trifurcation or quadrifurcation of the CHA, because it has been demonstrated that this is a risk factor for the development of gastric ulceration due to nontarget embolization during RE [36].

It is important to note that the recommended treatment strategies reflect our opinion, outlining the need for individualized RE strategies, and may need further tailoring towards the individual patient's case when used in clinical practice, to adjust for variants that could not be included in the extended anatomical classification, such as variants in the origin of extrahepatic branches or the presence of parasitized extrahepatic arteries.

\section{Conclusions}

The hepatic arterial configuration and segmental vascularization pattern show marked individual variability beyond well-known classifications of anatomical variants, relevant to RE. Therefore, it is recommended to assess the individual hepatic arterial configuration and segmental vascularization on MDCT, before the pretreatment angiography. We developed an individualized RE treatment strategy based on an extended classification of variant hepatic arterial anatomy.

Conflict of interest Andor F. van den Hoven has no conflict of interest. Maarten S. van Leeuwen has no conflict of interest. Marnix G.E.H. Lam has no conflict of interest. Maurice A.A.J. van den Bosch has no conflict of interest. 


\section{References}

1. Michels NA (1966) Newer anatomy of the liver and its variant blood supply and collateral circulation. Am J Surg 112:337-347

2. Hiatt JR, Gabbay J, Busuttil RW (1994) Surgical anatomy of the hepatic arteries in 1000 cases. Ann Surg 220:50-52

3. Gruttadauria S, Foglieni CS, Doria C, Luca A, Lauro A, Marino IR (2001) The hepatic artery in liver transplantation and surgery: vascular anomalies in 701 cases. Clin Transplant 15:359-363

4. Koops A, Wojciechowski B, Broering DC, Adam G, KrupskiBerdien G (2004) Anatomic variations of the hepatic arteries in 604 selective celiac and superior mesenteric angiographies. Surg Radiol Anat 26:239-244

5. Abdullah SS, Mabrut JY, Garbit V et al (2006) Anatomical variations of the hepatic artery: study of 932 cases in liver transplantation. Surg Radiol Anat 28:468-473

6. Covey AM, Brody LA, Maluccio MA, Getrajdman GI, Brown KT (2002) Variant hepatic arterial anatomy revisited: digital subtraction angiography performed in 600 patients. Radiology 224:542-547

7. Couinaud C (1989) Surgical anatomy of the liver revisited: embryology. Masson, Paris 1989

8. Jin GY, Yu HC, Lim HS et al (2008) Anatomical variations of the origin of the segment 4 hepatic artery and their clinical implications. Liver Transpl 14:1180-1184

9. Healey JE, Schroy PC, Sorensen RJ (1953) The intrahepatic distribution of the hepatic artery in man. J Int Coll Surg 20:133-148

10. Uliel L, Royal HD, Darcy MD, Zuckerman DA, Sharma A, Saad NE (2012) From the angio suite to the gamma-camera: vascular mapping and 99mTc-MAA hepatic perfusion imaging before liver radioembolization-a comprehensive pictorial review. J Nucl Med 53:1736-1747

11. van den Hoven AF, Smits ML, de Keizer B, van Leeuwen MS, van den Bosch MA, Lam MG (2014) Identifying aberrant hepatic arteries prior to intra-arterial radioembolization. Cardiovasc Intervent Radiol. doi:10.1007/s00270-014-0845-x

12. Wang S, He X, Li Z et al (2010) Characterization of the middle hepatic artery and its relevance to living donor liver transplantation. Liver Transpl 16:736-741

13. Suzuki T, Nakayasu A, Kawabe K, Takeda H, Honjo I (1971) Surgical significance of anatomic variations of the hepatic artery. Am J Surg 122:505-512

14. Winter TC 3rd, Freeny PC, Nghiem HV et al (1995) Hepatic arterial anatomy in transplantation candidates: evaluation with three-dimensional CT arteriography. Radiology 195:363-370

15. Erbay N, Raptopoulos V, Pomfret EA, Kamel IR, Kruskal JB (2003) Living donor liver transplantation in adults: vascular variants important in surgical planning for donors and recipients. AJR Am J Roentgenol 181:109-114

16. Winston CB, Lee NA, Jarnagin WR et al (2007) CT angiography for delineation of celiac and superior mesenteric artery variants in patients undergoing hepatobiliary and pancreatic surgery. AJR Am J Roentgenol 189:W13-W19

17. Saba L, Mallarini G (2011) Anatomic variations of arterial liver vascularization: an analysis by using MDCTA. Surg Radiol Anat 33:559-568

18. Onishi H, Kawarada Y, Das BC et al (2000) Surgical anatomy of the medial segment (S4) of the liver with special reference to bile ducts and vessels. Hepatogastroenterology 47:143-150
19. Couinaud C (1957) Le Foie Etudes anatomiques et chirurgicales. Masson, Paris

20. Gans HG (1955) Introduction to hepatic surgery. Elsevier, Amsterdam

21. Hortsjo CH (1951) The topography of the intrahepatic duct systems. Acta Anat 11:599-615

22. Fainsinger $M H$ (1950) The radiology of the intrahepatic biliary tract. S Afr J Med Sci 15:51-66

23. Elias H, Petty D (1952) Gross anatomy of the blood vessels and ducts within the human liver. Am J Anat 90:59-111

24. Bismuth H (1982) Surgical anatomy and anatomical surgery of the liver. World J Surg 6:3-9

25. Kennedy A, Coldwell D, Sangro B, Wasan H, Salem R (2012) Radioembolization for the treatment of liver tumors general principles. Am J Clin Oncol 35:91-99

26. Kao YH, Hock Tan AE, Burgmans MC et al (2012) Image-guided personalized predictive dosimetry by artery-specific SPECT/CT partition modeling for safe and effective $90 \mathrm{Y}$ radioembolization. J Nucl Med 53:559-566

27. Strunk H, Stuckmann G, Textor J, Willinek W (2003) Limitations and pitfalls of Couinaud's segmentation of the liver in transaxial imaging. Eur Radiol 13:2472-2482

28. van Leeuwen MS, Noordzij J, Fernandez MA, Hennipman A, Feldberg MA, Dillon EH (1994) Portal venous and segmental anatomy of the right hemiliver: observations based on threedimensional spiral CT renderings. AJR Am J Roentgenol 163:1395-1404

29. Fasel JH, Gailloud P, Grossholz M, Bidaut L, Probst P, Terrier F (1996) Relationship between intrahepatic vessels and computergenerated hepatic scissurae: an in vitro assay. Surg Radiol Anat 18:43-46

30. Ohashi I, Ina H, Okada Y et al (1996) Segmental anatomy of the liver under the right diaphragmatic dome: evaluation with axial CT. Radiology 200:779-783

31. Rieker OMP, Hintze C, Shunk K, Otto G, Thelen M (2000) Segmentanatomie der Leber in der Computertomographie: Lokalisieren wir die Läsionen richtig. Fortschr Röntgenstr 172:147-152

32. Louie JD, Kothary N, Kuo WT et al (2009) Incorporating conebeam $\mathrm{CT}$ into the treatment planning for yttrium-90 radioembolization. J Vasc Interv Radiol 20:606-613

33. van den Hoven AF, Prince JF, Samim M, Arepally A, Zonneberg BA, Lam MG, van den Bosch MA (2013) Posttreatment PET-CTconfirmed intrahepatic radioembolization performed without coil embolization, by using the antireflux surefire infusion system. Cardiovasc Intervent Radiol. doi:10.1007/s00270-013-0674-3

34. Abdelmaksoud MH, Louie JD, Kothary N et al (2011) Consolidation of hepatic arterial inflow by embolization of variant hepatic arteries in preparation for yttrium-90 radioembolization. J Vasc Interv Radiol 22(1364-1371):e1

35. Bilbao JI, Garrastachu P, Herraiz MJ et al (2010) Safety and efficacy assessment of flow redistribution by occlusion of intrahepatic vessels prior to radioembolization in the treatment of liver tumors. Cardiovasc Intervent Radiol 33:523-531

36. Lam MG, Banerjee S, Louie JD et al (2013) Root cause analysis of gastroduodenal ulceration after yttrium-90 radioembolization. Cardiovasc Intervent Radiol 36(6):1536-1547 